\title{
Track Processing Approach for Bearing-Only Target Tracking
}

\author{
Hui Chen \\ School of Electrical \& Information Engineering, Lanzhou University of Technology, Lanzhou, china \\ Email:jdch220@sina.com \\ Chen Li \\ Institute of Synthetic Automation, Xi'an Jiaotong University, Xi’an, china \\ Email: lynnlc@126.com
}

\begin{abstract}
This paper mainly studies angle-measurement based track processing approach to overcome the existing problems in the applications of traditional approaches for bearing-only target locating and tracking system. First, this paper gives suited data association algorithms including track initiation and point-track association. Moreover, a new tracking filtering association gate method is presented through analysis of the target motion characteristics in polar coordinates for improving bearing-only measurement confirming efficiency of real target and limiting false track overextension with the dense clutter. Then, by analyzing the feasibility of using multi-model technology, the IMM is adopt as filtering algorithm to solve existing problem in bearing-only tracking for complicated target motion in two dimensional angle plane. As the results, the two dimensional bearing-only tracking accuracy of real target is improved and false tracking is greatly limited. Moreover, computation cost of IMM is analyzed in view of the real-time demand of bearingonly tracking. Finally, this paper gives some concrete summary of multi-model choosing principle. The application of the proposed approach in a simulation system proves its effectiveness and practicability.
\end{abstract}

Index Terms - data association, multi-model filter, bearingonly tracking, passive sensor, targets

\section{INTRODUCTION}

The bearing-only target tracking using passive sensor means to obtain target motion element through angle-only measurement. Even if the target motion is constant velocity, namely $\mathrm{CV}$, the target motion is very difficult to express by using linear equation in the $\theta-\phi$ (azimuth and pitching) plane. This problem is high nonlinear in substance and target motion takes on higher maneuverability in polar plane. As yet, resolving method of this issue has been difficult in maneuvering target tracking domain [1-5]. Traditional multi-station joint passive tracking watching system estimates 3D information based on 2D measurement. Namely, this system indirectly gets the target position information by

Manuscript received January 16, 2009; revised June 21, 2009; accepted July 12, 2009. using multi-angle crossing approach [6,7]. But this approach brings more "ghosting" so that great computation cost is produced in target association tracking because of sensor measuring error itself. At the same time, this approach is limited by distance demand between the station and the other station. Thus, the target tracking error is enlarged and application value of this approach is little. In view of the demands of the concealment and flexibility for passive tracking system, the suited target tracking algorithm based on single passive sensor is urgent very much. In correlative references[8,9], some scholar make linear assumption for bearing-only target motion if sampling time of passive sensor is shorter and target confirming accuracy is high enough. Based on this assumption, azimuth angle and pitching angle of target is regarded as decoupling. From its inspiration, Aiming at the bearing-only tracking characteristics, a resolving approach completely based on angle-only measurements is studied by the deeply analysis of data association, association gate technology and multi-model filtering approach in this paper.

\section{TRACK INITIATION BASED ON BEARING-ONLY MEASUREMENTS}

\section{A. Existing problem and solution}

Multi-target track initiation is primary problem of maneuvering target tracking. It is a decision-making link of new target file establishment. For multi-target tracking processing, the right track initiation is key to reduce the burden of track processing and improve maneuvering target tracking effect. Traditional track initiation approach is difficult to find real target quickly and effectively. Taking into account the relevant characteristics of single passive tracking system and high real-time demand in the battlefield, mostly maneuvering target tracking need feedback target track information timely for tracking with scanning system. So taking sequential processing approach, which including heuristic algorithm and logic-based algorithm [10-13], is primary choice for track initiation in the bearing-only tracking system due to good real-time performance. It is worth noting that heuristic algorithm has high false track probability for simple heuristic rule. For high confirming efficiency and excellent performance, logic-based 
algorithm is widely adopted in real engineering application. This paper presents bearing-only track initiation approach based on traditional logic-based algorithm. It is depicted as

1) Isolated bearing-only measurement is used to establish new candidate track. By angular velocity rule that will be depicted as association gate design in part IV, measurements in next cycle are put into association decision.

2) Candidate track from step 1 is linearly extrapolated. Regard extrapolative point as associated center and make association decision for bearing-only measurements from next cycle. If some candidate track isn't associated with every measurement, those candidate tracks are terminated.

3) For every candidate track including three or more measurements, it is extrapolated by using second-order polynomial. Then make relevant associated decision according to the extrapolative point. The rest can be deduced up to the $N$-th step by analogy. Confirm target track by comparing the relation between innovation and threshold at last. For time $k(k=N)$, bearing-only measurement sequence corresponding to candidate track $m$ is

$$
\left\{z_{1, \rho(1, m)}, z_{2, \rho(2, m)}, \ldots, z_{N, \rho(N, m)}\right\}
$$

where $\rho(k, m)$ is depicted as measurement number corresponding to temporary track $m$.

Define cumulative innovation as

$$
J^{*}(m)=\sum_{k=1}^{N}\left[z_{k, \rho(k, m)}-\hat{z}_{k, m}\right]^{T}\left(R_{k}\right)^{-1}\left[z_{k, \rho(k, m)}-\hat{z}_{k, m}\right]
$$

where $\hat{z}_{k, m}$ is position estimation of candidate track through polynomial fitting, namely

$$
\hat{z}_{k, m}=\sum_{j=0}^{n_{x}} \hat{a}_{j}^{m}(k \Delta T)^{j} / j !
$$

where $\hat{a}_{j}^{m}$ is polynomial fitting coefficient.

Proved in reference [14], statistical value $J^{*}(m)$ is chi-square $\left(\chi^{2}\right)$ distribution with $N n_{z}-n_{x}$ degree of freedom ( $n_{x}$ is polynomial fitting order). If statistical value $J^{*}(m)$ satisfy test of threshold $\gamma$, which is gotten based on $\chi^{2}$ distribution with $N n_{z}-n_{x}$ degree of freedom, namely

$$
J^{*}(m) \leq \gamma
$$

Then, this candidate track is target track. It turn into tracking phase. For quick track initiation, this paper set continuous decision cycle as 4 .

\section{FEASIBILITY STUDY OF BEARING-ONLY DATA ASSOCIATION ALGORITHM}

\section{A. Nearest Neighbor Data Association (NNDA)}

NNDA $[15,16]$ is a early simple association algorithm. It helps to ensure the real-time demand of passive sensor target tracking. This approach associates the nearest neighbor measurement away from the tracking target in statistical view. This statistical distance is defined as weighed norm of innovation vector's , that is

$$
d_{k}^{2}=\tilde{\mathbf{z}}_{k \mid k-1}^{T} \boldsymbol{S}_{k}^{-1} \tilde{\mathbf{z}}_{k \mid k-1}
$$

where $\tilde{\mathbf{z}}_{k \mid k-1}$ is filter innovation, $\boldsymbol{s}_{k}$ is innovation covariance matrix, $d_{k}^{2}$ is norm of error vector.

The radical meaning of NNDA is uniquely choosing the nearest measurement away from target as associated object to estimate target state. For easy realization and little computing cost, it is applied to the tracking systems which have the high $S N R$ and the little target density. But when the measurement density is tremendous or multitarget association gate intercrossing each other, the nearest measurement not always come from the tracking target. Therefore, NNDA's anti-jamming capability is not so good and this approach easily brings false association.

\section{B. Joint Probabilistic Data Association(JPDA)}

JPDA [17-19] is advancing extending algorithm from PDA [20] (Probabilistic Data Association). It resolves the bug of false tracking in the application of PDA algorithm in the high maneuvering target density environment. This algorithm always is considered as one of the most perfect association approach. But comparatively, its computing cost is high because the association hypothesis event number between target and measurement is exponential expanded. Moreover, the distance between the two targets is very near. It is possible to bring bias and aggregation of track. Flow chart of this algorithm is shown in figure 1. The key technology of this algorithm lists the following

$$
\begin{aligned}
& \text { 1) Association gate } \\
& A^{t}(k) \equiv\left\{\frac{z}{\left[z-\widehat{z}^{t}(k \mid k-1)\right] S^{t}(k)^{-1}\left[z-\hat{z}^{t}(k \mid k-1)\right]^{\prime}} \leq g_{t}^{2}\right\}
\end{aligned}
$$

Where, $t=1,2, \ldots, N, \hat{Z}^{t}(k \mid k-1)$ is the position estimation of target $t$ at $k$; $S^{t}(k)$ is error covariance matrix of the measurement $\mathrm{t}$ at $k$. 


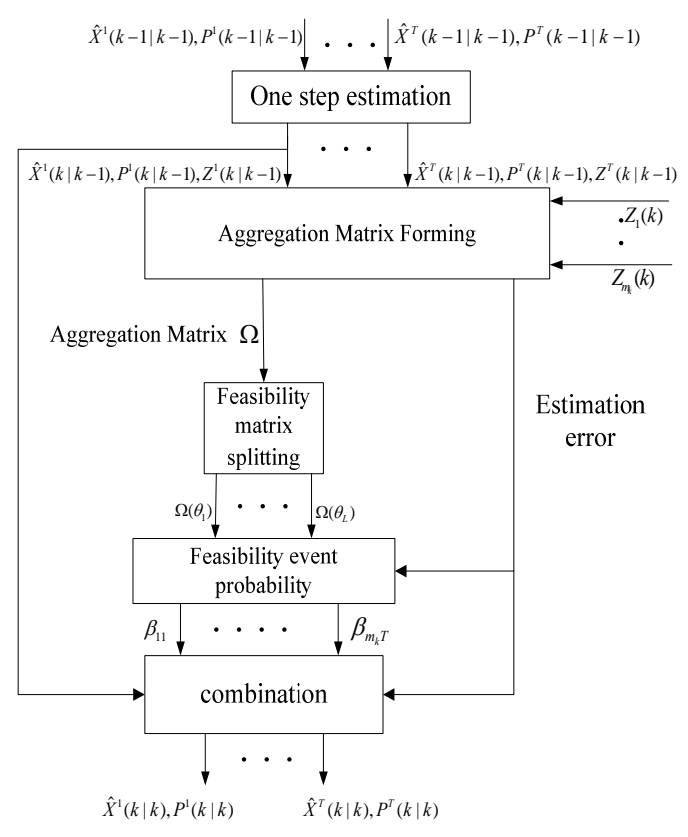

Figure 1. JPDA algorithm flow chart

$$
S^{t}(k)=E\left\{\left[z-\hat{z}^{t}(k \mid k-1)\right]\left[z-\hat{z}^{t}(k \mid k-1)\right]^{\prime}\right\}
$$

where $g_{t}^{2}$ denotes gate value.

If and only if the target measurement involves in the target association gate, it is regarded as valid measurement, or rejected. Assume that there are $m_{k}$ valid measurements involving in $N$ target-gates. Those measurements in the association gate are regarded as following association standby.

2) Clustering matrix

If consider that clustering matrix (or confirmed matrix) have $m_{k}$ rows and $N+1$ lines, clustering is defined as the most aggregate of conjoint tracking gate. All of targets are divided into different groups according to different clustering. There is a binary element matrix of clustering matrix which associates each of these groups all the time. Clustering matrix is defined as follows:

$$
\Omega=\left(\omega_{j t}\right), \quad j=1,2, \ldots, m_{k} ; t=0,1, \ldots, N
$$

where $\omega_{j t}$ denotes that measurement $j$ whether or not is contained by association gate.

$$
\omega_{j t}= \begin{cases}1 & \text { If } Z_{j}(k) \text { is in the association gate } \\ 0 & \text { or else }\end{cases}
$$

where $j=1,2, \ldots, m_{k}, t=1,2, \ldots, N$, and $t=0$ means that no target exist and correspondingly all line elements of $\Omega$ are 1 . In this time, any measurement originates from clutter or falsehood.

3) Feasibility Event

Feasibility event is produced by clustering matrix. Assume association event

$$
\theta_{j t}=\left\{\text { valid measurement } Z_{j}(k) \text { comes from the target } t\right\}
$$

where $j=1, \ldots, m_{k}, t=1,2, \ldots, N$.

When $t=0, \theta_{j 0}$ denotes measurement $Z_{j}(k)$ coming from clutter or noise. Note association event posterior probability

$$
\beta_{j t}=P\left\{\theta_{j t} \mid Z^{k}\right\}
$$

$B_{j t}$ is association probability. It is probability of association event appearance.

Define joint association event

$$
\theta=\bigcap_{j=1}^{m_{k}} \theta_{j} t_{j}
$$

Joint association event $\theta$ may represent matrix:

$$
\hat{\Omega}(\theta)=\left[\hat{\omega}_{j t}(\theta)\right]
$$

where

$$
\hat{\omega}_{j t}(\theta)=\left\{\begin{array}{lc}
1 & \theta_{j t} \subset \theta \\
0 & \text { or else }
\end{array}\right. \text {, }
$$

If satisfying two conditions as follows, joint association is defined as feasibility event $\theta$ :

- Every measurement only comes from a headspring, target or clutter, namely:

$$
\tau_{j}(\theta)=\sum_{t=0}^{T} \hat{\omega}_{j t}(\theta)=1 \quad j=1,2, \ldots, m_{k}
$$

- Every target only has a measurement, that is

$$
\delta_{t}(\theta)=\sum_{j=1}^{m_{k}} \hat{\omega}_{j t}(\theta) \leq 1 \quad t=1,2, \ldots, N
$$

For feasibility event $\theta$, corresponding matrix $\hat{\Omega}(\theta)$ is referred to as feasibility matrix. It is gotten by splitting clustering matrix, that is scanning $\Omega$ and only choosing a " 1 " in every row as feasibility matrix element. Except for the first line, every line of feasibility matrix only has a " 1 ". $\delta_{t}(\theta)$ is defined as target detecting indicator and $\tau_{j}(\theta)$ is defined as measurement indicator. Then clutter number is:

$$
\Phi(\theta)=\sum_{j=1}^{m_{k}}\left[1-\tau_{j}(\theta)\right]
$$

4) Feasibility Event Probability and Association Probability Calculation

The sum of joint association event is $L$ at $k$. Condition Probability, that is

- If clutter model is Poisson distribution, then

$$
P\left(\theta_{i} \mid Z^{k}\right)=\frac{\lambda^{\Phi}}{c^{\prime}} \prod_{j=1}^{m_{k}}\left[N_{t j}\left(Z_{j}(k)\right)\right]^{\tau} j \prod_{t=1}^{T}\left(P_{D}^{t}\right)^{\delta_{t}}\left(1-P_{D}^{t}\right)^{1-\delta_{t}}
$$

- Else if clutter model is uniformity distribution, then 


$$
P\left(\theta_{i} \mid Z^{k}\right)=\frac{1}{c_{v}} \frac{\Phi !}{\Phi} \prod_{j=1}^{m_{k}}\left[N_{t_{j}}\left(Z_{j}(k)\right)\right]^{\tau_{j}} \prod_{t=1}^{T}\left(P_{D}^{t}\right)^{\delta_{t}}\left(1-P_{D}^{t}\right)^{1-\delta_{t}}
$$

Where, c and c' are unitary element.

$$
N_{t_{j}}\left(Z_{j}(k)\right)=N\left(Z_{j}(k) ; Z_{j}^{t}(k \mid k-1), S_{j}^{t}(k)\right)
$$

Where, $N$ denotes normal distribution.

Finally, association probability is:

$$
\beta_{j t}=\sum_{i=1}^{L} P\left\{\theta_{i} \mid Z^{k}\right\} \hat{\omega}_{j t}\left(\theta_{i}\right)
$$

Where, $j=1, \ldots, m_{k} ; t=1, \ldots, T$

The probability of invalid measurement originating from target $t$ is:

$$
\beta_{0 t}=1-\sum_{j=1}^{m_{k}} \beta_{j t}, \quad j=1, \ldots, m_{k} ; t=1, \ldots, T
$$

\section{5) State Estimation}

$$
\hat{X}^{t}(k \mid k)=\sum_{j}^{m_{k}} \beta_{j t} \hat{X}_{j}^{t}(k \mid k)
$$

\section{AsSOCIATION GATE DESIGN FOR BEARING-ONLY TARGET TRACKING}

Gate technology is a key for data association. It affects the computing complexity of data association. Moreover, it decides the efficiency and performance of data association. The traditional gate technology is inefficient in $\theta$ - $\phi$ plane. They not only can not limit false track overextension with the dense clutter but also hardly confirm those real target tracks.

Firstly, initial gate will be design to confirm isolated measurement. There are two ordinary initial gate technologies. One is $\chi^{2}$ test method, the other is based on utmost velocity as $v_{k} \in\left[v_{\min }, v_{\max }\right]$. For the reason of complicated 2D bearing-only target motion as high nonlinear, the traditional gate technology is inefficiency. So gate technology in $\theta-\phi$ plane must be based on the characteristic of bearing-only target motion. Obviously, target motion is nearer, angle measurement change is larger. Thus, the area of confirming measurement must have good adaptability. In view of these factors, rational gate design is core for track processing in bearing-only tracking. Under normal circumstances, tracking system require that target will be reliably tracked at $D_{\text {min }}$ kilometers away from observation point. Design initial gate as the rule

$$
\left\|\frac{\mathbf{z}_{i}(k+1)-\mathbf{z}_{j}(k)}{t_{k+1}-t_{k}}\right\| \leq \frac{v_{\max }}{D_{\min } * 10^{3}}
$$

where $t_{k}$ is $k$-th sample time.

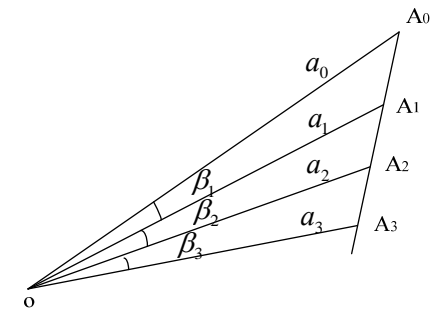

Figure 2. Angle change of target motion

Combining with the angle change analysis of bearing-only target motion, the gate of extrapolating by using linearly and second-order polynomial will be presented. As shown in figure 2, suppose that observation point is $\mathrm{O}$ and target moves along $A_{0} A_{3}$ as straight line. The sample points of the moving target at every sample time are $A_{0}, A_{1}, A_{2}, A_{3} \ldots$ and the distances of the different sample points away from observation point are $a_{0}, a_{1}, a_{2}, a_{3} \ldots$. The angles between observation point and the sample points are $\beta_{1}, \beta_{2}, \beta_{3} \ldots$. With a view to the assumption that sampling time of passive sensor is shorter and target confirming accuracy is high enough, so the average velocity of adjacent sampling circle is approximately equal and target motion is an approximately straight line form. Simultaneity, angle change in $\theta-\varphi$ plane is small. If supposing that $A_{2}$ corresponds to the sampling time $k$, equations are established based on triangular relationship according to figure 2 . Simplify those equations and get result as

$$
\frac{\Delta t_{k-2}^{k-1} \cdot a_{2}}{\Delta t_{k-2}^{k} \cdot a_{1}}=\frac{\sin \beta_{1}}{\sin \left(\beta_{1}+\beta_{2}\right)} \approx \frac{\beta_{1}}{\beta_{1}+\beta_{2}}
$$

where $\Delta t_{i}^{j}$ denotes time interval between sample time $i$ and sample time $j$. Then

$$
\begin{aligned}
& \left(\beta_{2}-\frac{\beta_{1}}{\Delta t_{k-2}^{k-1}} \cdot \Delta t_{k-1}^{k}\right) \approx\left[\frac{\Delta t_{k-2}^{k}}{\Delta t_{k-2}^{k-1}} \cdot \frac{a_{1}}{a_{2}}-\frac{\Delta t_{k-1}^{k}}{\Delta t_{k-2}^{k-1}}-1\right] \cdot \beta_{1} \\
& \leq\left(\frac{\Delta t_{k-2}^{k}}{\Delta t_{k-2}^{k-1}} \cdot \frac{D_{\text {min }}}{D_{\text {min }}-v_{\text {max }} \cdot \Delta t_{k-1}^{k}}-\frac{\Delta t_{k-1}^{k}}{\Delta t_{k-2}^{k-1}}-1\right) \cdot \beta_{1}
\end{aligned}
$$

Formula 26 denotes the upper limit of angle deviation when candidate track is linearly extrapolated in $\theta-\varphi$ plane. Use the upper limit as linearly extrapolating association gate in the course of track initiation. Make the assumption that measurement $\mathbf{z}_{i}(k)$ and the $j$-th component of vector $\hat{\mathbf{z}}_{m}^{L}(k)$ which is gotten by extrapolating candidate track $m$. If satisfying the following formula, measurement $\mathbf{z}_{i}(k)$ will be confirmed.

$$
\begin{aligned}
& \left|z_{i, j}(k)-\hat{z}_{m, j}^{L}(k)\right| \leq \mid\left(\frac{\Delta t_{k-2}^{k}}{\Delta t_{k-2}^{k-1}} \cdot \frac{D_{\min }}{D_{\min }-v_{\max } \cdot \Delta t_{k-1}^{k}}-\frac{\Delta t_{k-1}^{k}}{\Delta t_{k-2}^{k-1}}-1\right) \\
& \left(z_{k-1, i}-z_{k-2, i}\right) \mid
\end{aligned}
$$

When extrapolating candidate track using secondorder polynomial, suppose that $A_{3}$ corresponds to the sampling time $k$. Then, equations are established based on triangular relationship. Simplify those equations and get result as 


$$
\frac{\Delta t_{k-2}^{k-1} a_{3}}{\Delta t_{k-2}^{k} a_{2}}=\frac{\sin \beta_{2}}{\sin \left(\beta_{2}+\beta_{3}\right)} \approx \frac{\beta_{2}}{\beta_{2}+\beta_{3}}
$$

Then

$$
\begin{aligned}
& \beta_{3}-\left[\left(\frac{\beta_{2}}{\Delta t_{k-2}^{k-1}}+\Delta t_{k-2}^{k-1} \cdot \frac{\frac{\beta_{2}}{\Delta t_{k-2}^{k-1}}-\frac{\beta_{1}}{\Delta t_{k-3}^{k-2}}}{\Delta t_{k-3}^{k-1}}\right) \cdot \Delta t_{k-1}^{k}+\right. \\
& \left.\frac{\frac{\beta_{2}}{\Delta t_{k-2}^{k-1}}-\frac{\beta_{1}}{\Delta t_{k-3}^{k-2}}}{\Delta t_{k-3}^{k-1}} \cdot\left(\Delta t_{k-1}^{k}\right)^{2}\right]=\left(\frac{a_{2} \cdot \Delta t_{k-2}^{k}}{a_{3} \cdot \Delta t_{k-2}^{k-1}}-\frac{\Delta t_{k-1}^{k}}{\Delta t_{k-2}^{k-1}}-\frac{\Delta t_{k-1}^{k}}{\Delta t_{k-3}^{k-1}}-\right. \\
& \left.\frac{\left(\Delta t_{k-1}^{k}\right)^{2}}{\Delta t_{k-2}^{k-1} \cdot \Delta t_{k-3}^{k-1}}-1\right) \cdot \beta_{2}+\frac{\Delta t_{k-1}^{k} \cdot \Delta t_{k-2}^{k-1}+\left(\Delta t_{k-1}^{k}\right)^{2}}{\Delta t_{k-3}^{k-1} \cdot \Delta t_{k-3}^{k-2}} \cdot \beta_{1} \\
& \leq\left(\frac{\Delta t_{k-2}^{k}}{\Delta t_{k-2}^{k-1}} \cdot \frac{D_{\min }}{D_{\min }-v_{\max } \cdot \Delta t_{k-1}^{k}}-\frac{\Delta t_{k-1}^{k}}{\Delta t_{k-2}^{k-1}}-\frac{\Delta t_{k-1}^{k}}{\Delta t_{k-3}^{k-1}}-\frac{\left(\Delta t_{k-1}^{k}\right)^{2}}{\Delta t_{k-2}^{k-1} \cdot \Delta t_{k-3}^{k-1}}-1\right) \\
& \beta_{2}+\frac{\Delta t_{k-1}^{k} \cdot \Delta t_{k-2}^{k-1}+\left(\Delta t_{k-1}^{k}\right)^{2}}{\Delta t_{k-3}^{k-1} \cdot \Delta t_{k-3}^{k-2}} \cdot \beta_{1}
\end{aligned}
$$

Formula 28 denotes the upper limit of angle deviation when candidate track is extrapolated in $\theta-\varphi$ plane using second-order polynomial. Use the upper limit as second-order extrapolating association gate in the course of track initiation. If satisfying the following formula, measurement will be confirmed.

$$
\begin{aligned}
& \left|z_{i, j}(k)-\hat{z}_{m, j}^{R}(k)\right| \leq \mid\left(\frac{\Delta t_{k-2}^{k}}{\Delta t_{k-2}^{k-1}} \cdot \frac{D_{\min }}{D_{\min }-v_{\max } \cdot \Delta t_{k-1}^{k}}-\frac{\Delta t_{k-1}^{k}}{\Delta t_{k-2}^{k-1}}-\right. \\
& \left.\frac{\Delta t_{k-1}^{k}}{\Delta t_{k-3}^{k-1}}-\frac{\left(\Delta t_{k-1}^{k}\right)^{2}}{\Delta t_{k-2}^{k-1} \cdot \Delta t_{k-3}^{k-1}}-1\right) \cdot\left(z_{k-1, i}-z_{k-2, i}\right)+ \\
& \frac{\Delta t_{k-1}^{k} \cdot \Delta t_{k-2}^{k-1}+\left(\Delta t_{k-1}^{k}\right)^{2}}{\Delta t_{k-3}^{k-1} \cdot \Delta t_{k-3}^{k-2}} \cdot\left(z_{k-2, i}-z_{k-3, i}\right) \mid
\end{aligned}
$$

where $\hat{z}_{m, j}^{R}(k)$ is the $j$-th component of vector $\hat{\mathbf{z}}_{m}^{R}(k)$

which is gotten by extrapolating candidate track $m$ using second-order polynomial. These gate technologies make full advantage of the characteristic of bearing-only target motion and association gate adaptively adjust according to the measurements of the different candidate track.

\section{BEARING-ONLY TRACKING ALGORITHM BASED ON INTERACTIVE MULTI-MODEL(IMM)}

\section{A. Multi-model algorithm introduction}

In the course of study and engineering application of bearing-only maneuvering target tracking, the tracking performance of single model based adaptive filter isn't so good. Its limitation mainly is the competition between tracking accuracy and rapid response to target tracking. Especially to bearing-only tracking, for its higher target maneuverability and the variety of structure and parameter existing in target motion model, single-model adaptive filter is difficult to accurately recognize these varieties in time so that inaccurate model and false tracking appear.
Multi-model filter use several suited model to approximate the real target motion. Among them each model has a potential maneuvering mode. Random maneuvering of target is depicted as random hopping among models. By designing filter composed of several model, accordingly carry them into effectively execution for maneuvering target tracking. Thus, improving tracking performance is naturally shown. If making assumption that random hopping of target motion model state is discrete and target motion state is continuous, maneuvering target tracking is typical mixed estimation issue. Traditional solution of mixed estimation issue is combining estimation with decision-making. If making hard decision for the uncertain parameter and structure, the estimation result is usually bipolar optimization rather than global optimization. Under the circumstances, multi-model approach becomes mainly solution to mixed estimation nowadays.

The basic idea of multi-model maneuvering target tracking approach is mapping potential motion model into model set. Each model in set represents different maneuvering mode and varieties of models based filter works in parallel. State estimation output is Bayesian illation based data fusion of all filtering state estimations. Suppose that $i \in\left\{1,2, \ldots .,(\mathrm{Ms})^{k}\right\}$ is model state sequence index up to time $k$ and $M_{s}$ is model number in model set. Simultaneity, $\hat{x}_{i}(k \mid k)$ and $P_{i}(k \mid k)$ respectively is state estimation and error covariance under assumption of model state sequence $m^{k}$ matching model sequence $m_{i}^{k}$ of i index. $P\left\{m^{k}=m_{i}^{k} \mid z^{k}\right\}$ is posterior probability of this assumption. $S^{k}$ is a set of all potential model sequence. $Z^{k}$ is measurement sequence. Then, optimal multi-model estimation under LMSE need to considered all potential model state sequence assumption, namely

$$
\left\{\begin{array}{l}
\hat{x}(k \mid k)=\sum_{m_{i}^{k} \in S^{k}} \hat{x}_{l}(k \mid k) P\left\{m^{k}=m_{i}^{k} \mid Z^{k}\right\} \\
\mathrm{P}(k \mid k)=\sum_{m_{i}^{k} \in S^{k}}\left\{P_{i}\left(k \mid k+\left(\hat{x}(k \mid k)-\hat{x}_{i}(k \mid k)\right)\left(\hat{x}(k \mid k)-\hat{x}_{i}(k \mid k)\right)^{\prime}\right\} P\left\{s^{k}=m_{i}^{k} \mid z^{k}\right\}\right.
\end{array}\right.
$$

Apparently, the number of potential model sequence assumption presents an index growth with time flowing. It produces an unacceptable computation cost. Especially to the disadvantage that target threatening degree can't be estimated for distance information lack in passive tracking system, so real-time is very important to this system. Thus, the computation cost of selective approach must satisfy the demand of tracking operation. IMM algorithm [21-23] proposed by H.A.P.Bolm is an inferior optimized multi-model algorithm which has high cost-effective. This algorithm makes assumption that transformation of different model obeys finite Markov chain of known transition probability. It has the same performance as GBP2 and advantage of computation cost as GBP1. IMM is regarded as the first multi-model algorithm up to application value[24]. 
B. IMM algorithm based on bearing-only measurement

In polar coordinate, redefine state variable as

$$
X=\left[\begin{array}{llll}
\theta & \varphi & \dot{\theta} & \dot{\varphi}
\end{array}\right]^{\prime}
$$

Assume that target motion can be depicted as a model from $r$ assumption model in some time, note model set $M_{r}:=\{1, \ldots, r\}$. The effective event of model $j$ is noted as $M^{j}(k)$ in sampling period $\left(t_{k-1}, t_{k}\right]$. For the assumption model $j$, whose target state equation is

$$
\left\{\begin{array}{l}
X(k)=F^{j}(k-1) X(k-1)+G^{j}(k-1) W^{j}(k-1) \\
Z(k)=H^{j}(k) X(k)+V^{j}(k)
\end{array}\right.
$$

Assume that probability of model $j$

$$
\mu^{j}(0)=\operatorname{Pr}\left\{M^{j}(0)\right\}
$$

Transition probability

$$
p_{i j}=\operatorname{Pr}\left\{M^{j}(k) \mid M^{i}(k-1)\right\}
$$

It is known and decided by Markov chain from $M^{j}(k-1)$ to $M^{j}(k)$ in this time.

\section{1) Mixed probability calculating}

If $M^{j}(k)$ and measurement set $Z_{k-1}\left(Z_{k-1}:=\{Z(1), Z(2), \ldots, Z(k-1)\}\right)$ is known in sampling time $k$, appearance probability of $M^{i}$ can be expressed as $u^{i \mid j}(k-1 \mid k-1)=P\left\{M(k-1)=M_{i} \mid M(k)=M_{j}, Z_{k-1}\right\}=\frac{1}{c_{j}} p_{i j} u^{i}(k-1)$

Where, $i, j=1,2, \ldots, \mathrm{n}, c_{j}$ is normalization constant.

\section{1) Interacting and mixed calculating}

Give the calculating expression of $\hat{X}^{i}(k-1 \mid k-1)$ and corresponding covariance $P^{i}(k-1 \mid k-1)$ for different model

$$
\left\{\begin{aligned}
\hat{X}^{0 j}(k-1 \mid k-1)= & \sum_{i=1}^{n} \hat{X}^{i}(k-1 \mid k-1) u^{i j j}(k-1 \mid k-1) \\
P^{0 j}(k-1 \mid k-1)= & \sum_{i=1}^{n} u^{i l j}(k-1 \mid k-1)\left\{P^{i}(k-1 \mid k-1)+\left[\hat{X}^{i}(k-1 \mid k-1)-\right.\right. \\
& \left.\left.\hat{X}^{0 j}(k-1 \mid k-1)\right]\left[\hat{X}^{i}(k-1 \mid k-1)-\hat{X}^{0 j}(k-1 \mid k-1)\right]^{\prime}\right\}
\end{aligned}\right.
$$

\section{2) Model conditional filtering}

Regard the gotten mixed initial condition from step 2 and current measurement $Z(k)$ as input of each filter in time $k$. Thus, figure out newly model estimation, i.e. $\hat{X}^{j}(k \mid k)$ and $P^{j}(k \mid k)$. Together with predicted measurement $\hat{Z}^{j}(k \mid k-1)$ and corresponding innovation covariance $S^{j}(k)$, figure out likelihood function of filter

$$
\Lambda^{j}(k)=\frac{1}{\sqrt{2 \pi\left|S^{j}(k)\right|}} \exp \left\{-\frac{1}{2}\left[Z(k)-\hat{Z}^{j}(k \mid k-1)\right]^{\prime}\left(S^{j}(k)\right)^{-1}\left[Z(k)-\hat{Z}^{j}(k \mid k-1)\right]\right\}
$$

Where, function distribution is Gauss.

3) Renewing model probability

Each renewing model probability lists as follows

$$
u^{j}(k)=\frac{1}{c} \Lambda^{j}(k) \sum_{i=1}^{n} p_{i j} u^{i}(k-1)
$$

4) State and covariance estimation.

Formula of State and covariance estimation is

$$
\left\{\begin{array}{l}
\hat{X}(k \mid k)=\sum_{j=1}^{n} \hat{X}^{j}(k \mid k) u^{j}(k) \\
P(k \mid k)=\sum_{j=1}^{n} u^{j}(k)\left\{P^{j}(k \mid k)+\left[\hat{X}^{j}(k \mid k)-\hat{X}(k \mid k)\right]\left[\hat{X}^{j}(k \mid k)-\hat{X}(k \mid k)\right]^{\prime}\right\}
\end{array}\right.
$$

By analysis of algorithm framework, measurement information utilization of IMM exists in not only filtering estimation but also model probability. And IMM can adaptively adjust model by model probability change. Simultaneity, this algorithm has modularization characteristic. Through different application, filtering module can adopt all kinds of linear and nonlinear filtering algorithm. Finally, efficiency is improved in virtue of each filtering module working side by side in this algorithm.

\section{Model selection for bearing-only tracking}

In this paper, model selection only limits to $\mathrm{CV}$ and CA because there isn't so good performance for CT in polar coordinate and value of $\omega$ is difficult to grasp. The research indicates that common motion can be approximated by certain combination of CV and CA.

\section{SimULATION}

For getting more clear result, track initiation will be simulated firstly in order to test its distinguishing ability and fast ability in clutter environment. At last, set environment to test the final effect of track processing system as a whole.

\section{A. Track initiation simualtion}

Introduce guide line for simulation judgment [25].

1) False probability of track initiation (FP)

$$
F P=\sum_{i=1}^{N} f_{i} / \sum_{i=1}^{N} n_{i}
$$

where $N$ is simulation number based on MonteCarlo. $N=30$ in this paper. $f_{i}$ is false track number and $n_{i}$ is initiated track number as a simulation.

2) Correct initiation probability $\left(C_{j}\right)$

$$
C_{j}=\frac{\sum_{i=1}^{N} l_{i j}}{N}
$$


where $l_{i j}$ represents if target $j$ is initiated correctly in the $i$-th Monte-Carlo simulation. Correct is 1 , or 0 .

TABLE I. INITIAL STATES OF THE TARGETS IN CARTESIAN COORDINATE

\begin{tabular}{|c|c|c|c|c|c|c|}
\hline Target & $\begin{array}{c}\text { Process } \\
\text { noise } \\
\text { coefficient }\end{array}$ & Model & Velocity & X & Y & Z \\
\hline 1 & 0.70 & CV & 980 & 15000 & 5100 & 5000 \\
\hline 2 & 0.90 & CA & 520 & 10000 & 1200 & 1000 \\
\hline 3 & 0.60 & CV & 500 & 12000 & 100 & -800 \\
\hline 4 & 1.25 & CA & 430 & 9500 & 6000 & 200 \\
\hline 5 & 1.00 & CV & 480 & 10000 & 800 & -600 \\
\hline 6 & 0.70 & CV & 550 & 9000 & -2500 & 1000 \\
\hline 7 & 0.50 & CV & 530 & 15000 & 8000 & -600 \\
\hline
\end{tabular}

There are seven targets in simulation environment. Their movements are CA and CV respectively. Process noise is white Gaussian noise. Initial states of the targets list as table 1 (position and velocity unit are $\mathrm{m}$ and $\mathrm{m} / \mathrm{s}$ respectively.). There, acceleration of target 2 and target 4 are $75 \mathrm{~m} / \mathrm{s}^{2}$ and $87 \mathrm{~m} / \mathrm{s}^{2}$ respectively. Figure out bearingonly measurement in polar $(\theta-\phi)$ coordinate based on these target tracks in Cartesian coordinate. Assume clutter density $\lambda=6.25 * 10^{-5} / \mathrm{mrad}^{2}$ (Clutter number is Poisson distribution. Clutter is scattered as uniform distribution. ) and measurement noise coefficient is $1.5 \mathrm{mrad}$. Note that angle unit is one thousandth of radian, namely mrad. There, $D_{\min }=10 \mathrm{~km}, T=1.5 \mathrm{~s}, v_{\max }=1 \mathrm{~km} / \mathrm{s}$, $a_{\max }=100 \mathrm{~m} / \mathrm{s}^{2}$ and threshold test significance level $\alpha=0.01$. In order to test speed of track initiation, this simulation is based on 6 sample cycle. Typical clutter environment is shown in figure 3. Effect of track initiation is shown in figure 4. Based on 30 times MonteCarlo simulation, false track probability with different clutter density is shown in figure 5 . Correct track initiation probabilities of the targets as follow

$$
\begin{aligned}
& \mathrm{C}_{1}=86.7 \%, \mathrm{C}_{2}=83.3 \%, \mathrm{C}_{3}=93.3 \%, \mathrm{C}_{4}=80.0 \%, \\
& \mathrm{C}_{5}=80.0 \%, \mathrm{C}_{6}=90.0 \%, \mathrm{C}_{7}=93.3 \% .
\end{aligned}
$$

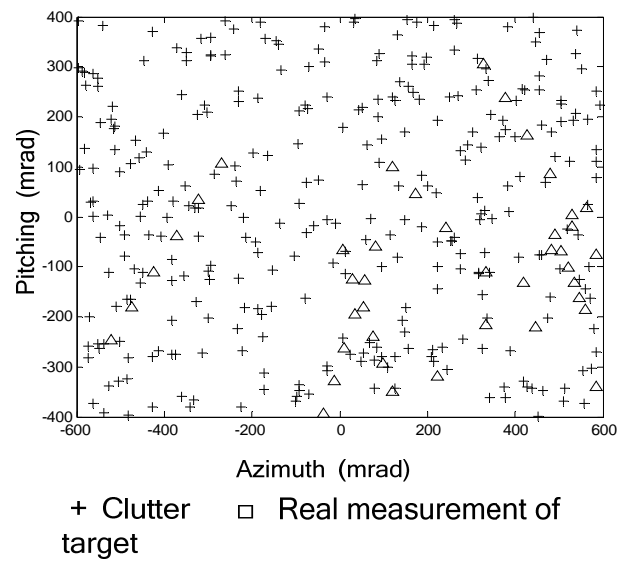

Figure 3. Bearings-only measurements in clutter environment

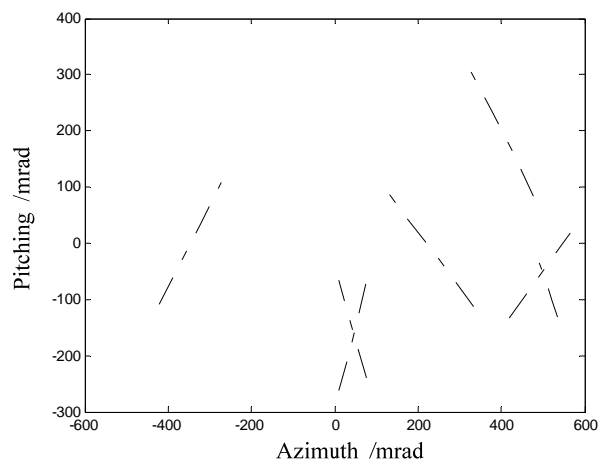

Figure 4. Effect of track initiations

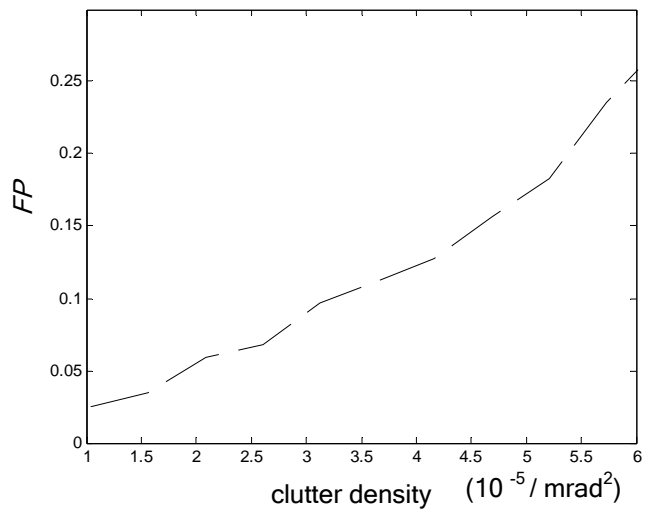

Figure 5. False track probabilities based on the clutter density

\section{B. The tracking effect based on JPDA-IMM for maneuvering target}

Here, a selective maneuvering target is shown in figure 6 and figure 7. The performance comparison of IMM and single model approach based on JPDA algorithm is shown in figure 8 and figure 9 based on Monte Carlo simulation. Where, make assumption that $T=1.5$ s, sampling number $N=350$.

Assume that measurement noise is Gauss white noise whose coefficient is 1 mrad. In this tracking filtering algorithm, the approach of direct angle modeling is chosen. In the single model algorithm, tracking model is $\mathrm{CV}$ whose state noise coefficient is $0.7 \mathrm{mrad}$. Simultaneity, there is an IMM filter composed of 4 model used to track. These models are depicted as: There are different $Q$ matrix for model 1-model 3, which is $\mathrm{CV}$ whose state noise coefficient respectively are $1,0.1,0.01$ and model 4 is chosen as CA whose state noise coefficient respectively is 0.1 . Initial model probability matrix

$$
\mu_{0}=\left[\begin{array}{llll}
1 / 4 & 1 / 4 & 1 / 4 & 1 / 4
\end{array}\right]
$$

Model transition probability matrix 


$$
P=\left[\begin{array}{llll}
0.97 & 0.01 & 0.01 & 0.01 \\
0.01 & 0.97 & 0.01 & 0.01 \\
0.01 & 0.01 & 0.97 & 0.01 \\
0.01 & 0.01 & 0.01 & 0.97
\end{array}\right]
$$

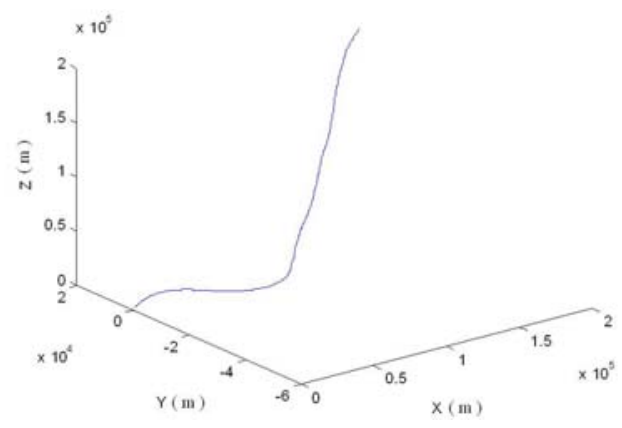

Figure 6. Target track in Cartesian coordinate

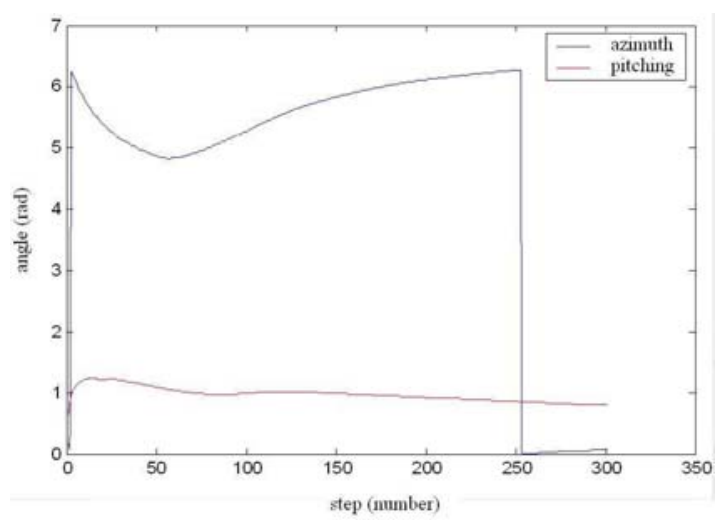

Figure 7. Target track in bearing-only coordinate

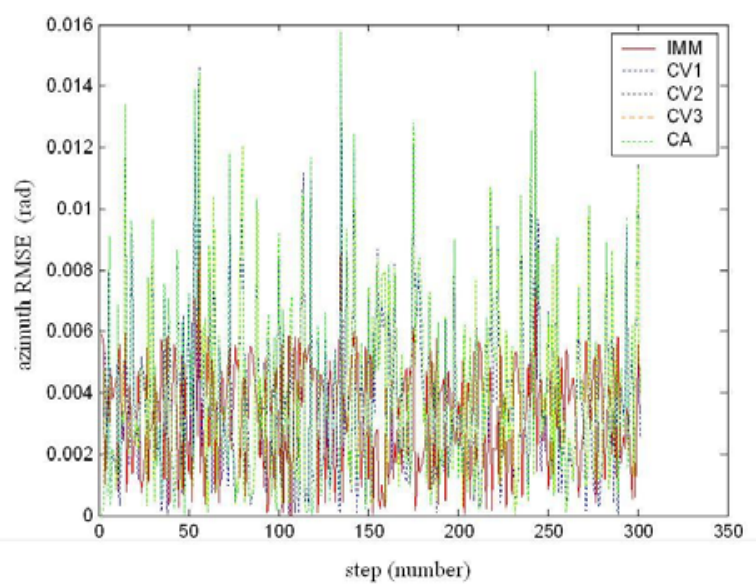

Figure 8. Azimuth RMSE comparison of IMM and single model

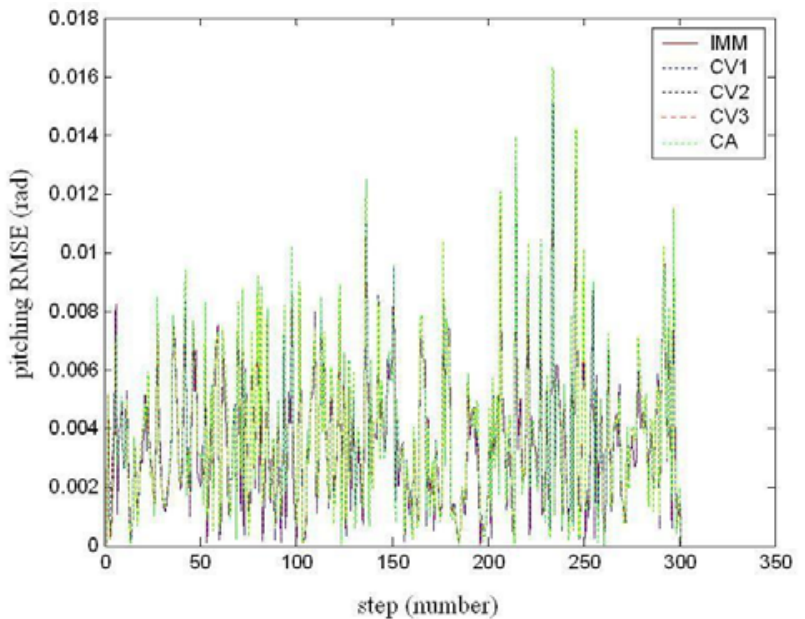

Figure 9. Pitching RMSE comparison of IMM and single model

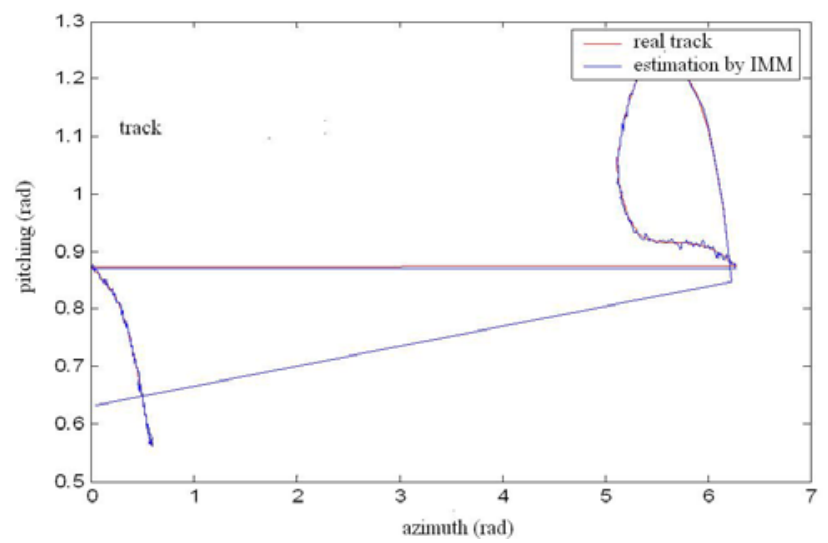

Figure 10. Tracking effect in bearing-only coordinate

In these simulation figures, tracking comparison of IMM and every model of composing IMM are given in polar coordinate. Paying more attention to the dimension, horizontal and vertical axis, respectively, are radian and RMSE. Simulation time cost is shown in Table 2.

TABLE II. COMPUTATION COST COMPARISON OF IMM AND

\begin{tabular}{|c|c|c|c|c|c|}
\hline Model & IMM & CV1 & CV2 & CV3 & CA \\
\hline Time(s) & 1.0940 & 0.2350 & 0.2030 & 0.2180 & 0.2500 \\
\hline
\end{tabular}

\section{CONCLUSION}

Simulation of track initiation approach in this paper shows that its confirming efficiency is effectively improved based on the presented gate technology. This approach effectively affirms the correct probability of track initiation for maneuvering target. According to simulation result of whole track processing system and application of actual ship-borne infrared system, adopting JPDA as data association is suitable for bearing-only 
tracking system using passive sensor. Whereas, its performance takes on not so good comparing with performance in Cartesian coordinate. Moreover, JPDA is very high need for target detecting probability, sampling time and clutter density etc. Unsuitable value might lead to missing tracking. There is also clear conclusion that tracking accuracy is improved by using IMM. On the other hand, algorithm complexity is enhanced in deed, and that computation cost is in direct proportion to model number. Under the accuracy condition satisfied, model number choice is less as possible. By simulation, draw a conclusion: if the model number of $\mathrm{CV}$ exceeds 3 or number of CA exceeds 2, minimal performance improvement is displayed in the bearing-only tracking with computation cost greatly increase. It is the reason that too much unnecessary model competition in the multi-model data fusion. Excessive use of the model, not only increase computation cost but also reduce the accuracy of estimation. Based on simulation and outdoor actual debug of ship-based defense system, balancing the two factors that accuracy and computation cost, that choose the two CV and one CA to compose of multimodel tracking filter can get the more satisfied results.

\section{REFERENCES}

[1] Popp R L, Pattipati K R, Bar-Shalom Y. m-Best S-D assignment algorithm with application to multitarget tracking[J]. IEEE Trans. on AC, 2001, 37 (1):22 - 38.

[2] Eli Fogel, Motti Gavish. Nth-order Dynamics Target Observability from Angle Measurements[J] . IEEE Trans. on AES, 1998, 3(24):305 - 307.

[3] Mangzuo S. Range Information Extraction from Tracking Data Using Object Kinematic Parameters[J] . SPIE, 1995, 2561: 484-488.

[4] Kronhamn T R. Bearings-only Target Motion Analysis Based on A Multi-hypothesis Kalman Filter and Adaptive Oweship Motion Control [R]. IEE Proc. Radar, Sonar Navigation, 1998, 145 (4): 247 - 252.

[5] Pattipati K R, Deb S, Bar-Shalom Y, et al. A new relaxation algorithm and passive sensor data association [J]. IEEE Trans. on AC, 1992, 37(2): 98-213.

[6] Somnath Deb, Murali Yeddanapudi, Krishna Pattipati, et al. A generalized S-D assignment approach for multisensormultitarget state estimation [J]. IEEE Trans. on AES, 1997, 33(2): 523-538.

[7] WANG Ming-hui, YOU Zhi-sheng, ZHAO Rong-chun, et al. An fast data association approach of passive sensors [J]. Electronic Journal, 2000, 28(12): 45-47.

[8] Ito M, Tsujimichi S, Kosuge Y. Sensor-to-sensor target association in a network of passive sensors[J]. 23rd International Conference on Industrial Electronics, Control and Instrumentation, 1997, (3): 1260-1264.

[9] PAN Li-na. A track initiation and filtering approach of shipborne infrared surveillance system in multi-target tracking [J].Electro-Optic Warfare \& Radar Passive Countermeasures, 1998, (2): 19-23.

[10] Grevera G J, Udupa J K. An objective comparision of 3-D image interpolation methods[J]. IEEE Transactions on Medical Imaging, 1998, 17(4): 642-652.

[11] Grevera G J, Udupa J K. Shape-based interpolation of multidimensional grey-level images[J]. IEEE Transactions on Medical Imaging, 1996, 15(6): 881-892.

[12] Hu Z J, Leung H. Statistical performance analysis of track initiation techniques[J]. IEEE Transactions on AES, 1997, 45(2): 445-456.
[13] A Goshtasby, D A Turner, L V Acekerman. Matching of tomographic slices for interpolation[J]. IEEE Transactions on Medical Imaging, 1992, 11(4): 507-516.

[14] Bar-shalom Y, Fortmann T E. Tracking and data association[M]. New York, 1988. 252 - 263.

[15] Singer R A, Sea R G. A new filter for optimal tracking in dense multi-target environment[C]. Proceedings of the ninth Allerton Conference Circuit and System Theory. Urbana-Champaign, USA: Univ. of Illinois, 1971. 201 211.

[16] Singer R A,Stein J J. An optimal tracking filter for processing sensor data of imprecisely determined origin in surveillance system[C]. Proceedings of the tenth IEEE Conference on Decision and Control. USA: Institute of Electrical and Electronics Engineers, 1971. 171 175.

[17] Roecker J A, Phillis G L. Suboptimal joint probabilistic data association[J]. IEEE Transactions on Aerospace and Electronic Systems, 1993, 29(2): 510 517.

[18] Roecker J A. A class of near optimal JPDA algorithm[J]. IEEE Transactions on Aerospace and Electronic Systems, 1994, 30(2): 504 510

[19] Fisher J L,Casasent D P. Fast JPDA multi-target tracking algorithm[J]. Applied Optics, 1989, 28(2): 371 376.

[20] Bar-Shalom Y, Tse E. Tracking in a cluttered environment with probabilistic data association[J]. Automatica, 1975, 11(9):451 460.

[21] Metropolis N, Rosenbluth A W, M N Rosenbiuth, etal. Equations of state calculations by fast computing machines. Journal of chemical phyisics, 1953, 21(6): 1087 1091.

[22] Kong A, Liu J S, Wong W H. Sequential imputations and Bayesian missing data problem.. Jounal of American Statistical Association, 1994, 89(425): 278 288.

[23] Gordon N J, Salmond D J, Smith A F M. Novel approach to nonlinear-non-gaussian Bayesian state estimation. IEEProceeding-F, 1993, 140(2):107 113.

[24] Johnston L A, Vikram Krishnamurthy. An Improvement to the Interacting Multiple Model (IMM) Algorithm. IEEE Trans. on Signal Processing, 2001, 49: 2909-2923.

[25] ZHU Hong-yan, HAN Chong-zhao, HAN Hong, et al. Study on Approaches for Track Initiation[J]. Acta Aeronautica et Astronautica Sinica, 2004, 25(3): 284-288.

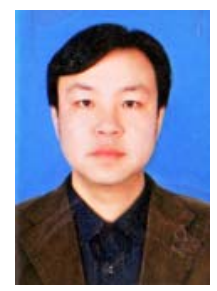

Hui Chen was born in Lanzhou, China in 1978. He received the B.Sc. and M.Sc. degrees from Lanzhou University of Technology and Xi'an Jiaotong University, in 2001 and 2007, respectively.

In 2001, he joined the School of Electrical \& Information Engineering at Lanzhou University of Technology at Lanzhou, in The China, as a Research Engineer. He spent the 2002-2003 academic year as a visiting scholar to study control theory at Tsinghua University. He currently has active collaborations with institute of synthetic automation, Xi'an jiaotong university. He has published over 10 refereed journal and conference papers in the areas of control systems and multisensor data fusion. His representative published articles lists as follow: Fast track initiation approach for passive sensor system (Chendu, China: Opto-Electronic Engineering, 2008), New Strategy of Improving Stream Control Transmission Protocol Performance over Satellite Link (Kunming, China: The 27th Chinese Control Conference, 2008), Track initiation algotithm for two-dimensional target 
tracking based on bearing-only measurement (Beijing, China: Acta Aeronautica Et Astronautica Sinica , 2009), etc. Moreover, he was a participant of some engineering projects such as control system of anode baking product line for BaiYing Aluminium Factory, bearing-only tracking system for Chinese Ship Industry Co, stage control system of the PLA General Political Department Opera House and so on. His activities currently focus on maneuvering target tracking based on passive sensor. His research interests include information fusion, estimation theory, and control theory.

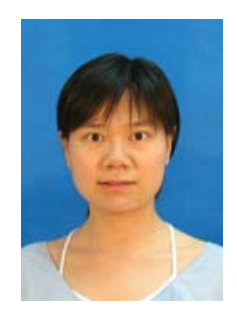

Chen Li was born on February 25, 1981. She received the B.Sc. and PH.D. degrees from Xi'an Jiaotong University, in 2003 and 2008, respectively.

In 2008, she joined the Xi'an Jiaotong University, School of software at Xi'an, in The China, as a Teacher. His representative published articles lists as follow: Data association for target tracking by IR sensors (Aircraft Engineering and Aerospace technology, 2007), A new smoothing approach with diverse fixedlags based on target motion model (International Journal of Automation and Computing, 2006), Data association for target tracking by several passive sensors (Canada: 2007 IEEE International Conference on Systems, Man and Cybernetics (SMC), 2007), etc. Her research interests include Bayesian estimation, accident risk assessment, and stochastic, discrete-event-controlled processes on hybrid state spaces. Especially, her researches about bearing-only target tracking have certain influence. 